\title{
Los megaeventos y sus consecuencias urbanas Posibles perspectivas hacia las futuras experiencias brasileñas
}

\author{
Ana Carla Cortes de Lira \\ Màster d'Antropologia Urbana, Universitat Rovira i Virgili \\ acclira@gmail.com
}

Resumen: Partiendo de la excusa de los campeonatos deportivos de ámbito mundial previstos en Brasil para los próximos años, el presente ensayo se propone discutir el papel de los megaeventos en la transformación social y urbanistica de sus ciudades-sede, tomando como principal referente los Juegos Olimpicos de Verano. A través de un abordaje bistórico, y con referencias a otros autores que se dedican al tema en cuestión, se plantea un breve repaso del conjunto de experiencias olimpicas de los últimos 20 años, de las cuales se entresacan las tendencias que hemos observado y la relación entre la evolución de los criterios de organización de dichos eventos y el modelo vigente de gestión de las ciudades. Como objetivo, se busca establecer un panorama a través del cual se puedan evaluar los posibles impactos de las próximas Olimpiadas, a saber, Londres 2012 y, más especificamente, Río de Janeiro 2016. Mientras la capital inglesa, a pocos días de su estreno como ciudad olimpica, concluye su etapa de preparación, en la antigua capital brasileña justo se empiezan a producir abora las divergencias $y$ conflictos sociales que configuran el proceso de organización de un evento de este calibre.

Palabras clave: Megaeventos, Olimpíadas, Juegos Olimpicos, gestión urbana, transformación urbanistica.

Abstract: With the excuse of the games that are shortly to be held in Brazil, this article aims to discuss the role of mega-events in general and summer Olympic Games in particular in the social and urban transformation of their host cities. It uses a historical approach and refers to other authors who have discussed the subject to review the Olympic experiences of the past 20 years, and highlight some of the trends and the relationship between the evolution of the criteria for organizing these events and the current model of city management. The aim is to enable future evaluations to be made of the potential impacts of the upcoming Olympic Games: London 2012 and, more specifically, Rio de Janeiro 2016. Whereas the capital of Britain, only a few days before its premiere as the Olympic city, has already concluded the preparation stage, the Brazilian capital is just beginning to become aware of the divergences and social conflicts that shape the process of organizing an event of this level.

Keywords: Mega-events, Olympics, Olympic Games, urban management, urban transformation. 


\section{Introducción}

El día 2 de octubre de 2009, en Copenhague (Dinamarca), la ciudad brasileña de Río de Janeiro resultó victoriosa en su segunda candidatura con vistas a albergar los Juegos Olímpicos de Verano. Tras un primer intento para las Olimpíadas de 2004 y una importante experiencia previa con los Juegos Panamericanos de 2007, finalmente la edición de las Olimpíadas de Verano de 2016 se llevará a cabo en una ciudad iberoamericana. Dos años antes de este anuncio ya había conquistado otra victoria similar, al ser elegido Brasil como el país que albergaría la Copa Mundial de Fútbol de 2014. Esta sucesión de éxitos constituye la entrada del país en el mundo de los megaeventos deportivos. Desde el punto de vista de los cambios urbanos, ¿qué significan tales logros en el contexto actual?

Partiendo de una mirada hacia las tendencias observadas en las Olimpíadas modernas, desde su creación hasta su edición inminente, Londres 2012, el presente ensayo, sin contestar a esta pregunta directamente, pretende ofrecer un panorama a través del cual se puedan apreciar los impactos (no) esperados en los Juegos brasileños.

Para tanto, en un primer momento se ofrecerá un breve y resumido repaso del creciente papel de los megaeventos deportivos como agentes de transformación urbanística, buscando destacar los aspectos más relevantes para la comprensión de su relación con el contexto político y económico mundial de cada momento histórico. La segunda parte presentará parámetros observados en experiencias olímpicas de los últimos 20 años, a partir de los cuales se pueden verificar tendencias comunes desde los puntos de vista físico, político, económico y social. Finalmente, la tercera parte del ensayo destacará algunos de los principales elementos del proyecto de Londres 2012 en la zona de East London, que sugieren posibilidades de cambios de actitud frente los retos afrontados por una ciudad olímpica en la actualidad. El presente esquema de análisis, todavía de carácter preliminar, tiene como premisa subsidiar el desarrollo futuro de un estudio más ampliado hacia otras posibilidades interpretativas. 


\section{Megaeventos y transformación de las ciudades}

A lo largo de la historia, la relación entre megaeventos y ciudad estuvo desde siempre caracterizada por una relación reciproca de beneficios, que incluyen el prestigio nacional y la proyección mundial de la ciudad sede en contrapartida al fortalecimiento de la "marca" del evento, a través de una correcta y bien estructurada organización. Así, la excusa de lo efímero se convirtió poco a poco en elemento propulsor de la construcción de monumentos, edificaciones, grandes equipamientos y realización de obras de infraestructura capaces de interferir en el paisaje y en las dinámicas urbanas hasta entonces establecidas.

Las Exposiciones Universales, realizadas desde 1851 y dedicadas principalmente a la exhibición de los últimos progresos de la industria, tuvieron como primera sede la ciudad de Londres - aunque en un primer momento todavía no se planteaban como un evento cíclico-. En dicha ocasión, el Reino Unido ocupaba el lugar de mayor potencia económica mundial, y su espíritu emprendedor estuvo representado por la construcción del Palacio de Cristal, proyectado por el arquitecto Joseph Paxton, primer edificio de la historia construido $100 \%$ con elementos prefabricados - hierro y cristal—, de montaje rápido y posibilidad de desmontaje, características que luego le convirtieron en un símbolo popular de modernidad y civilización. Con motivo de la organización de la Exposición — considerada entonces el mayor evento político, económico y social del mundo- se convirtió en una práctica habitual la urbanización de las zonas que abrigaban el evento, con la construcción de marcos visuales en la ciudad, a ejemplo de la edición de 1888 de Barcelona, en razón de la cual se promovió la construcción del Parque de la Ciutadella, la construcción del Arco del Triunfo, el Paseo Marítimo y el monumento a Cristóbal Colón, entre otras obras. Así como en ésta, la edición de 1929, que también se llevó cabo en Barcelona, fue aprovechada por sus organizadores como escaparate para proyectar la industria catalana y como una oportunidad de remodelación urbana. Ubicada en la montaña de Montjuïc, con vistas a la Exposición se realizó la urbanización de parte de esta zona, con la construcción del Palacio Nacional (hoy Museo Nacional de Arte de Cataluña), el pabellón Mies van der Rohe, la Fuente Mágica, la Plaza de España, el Teatro Grec, entre otros edificios. A través de la Exposición de 1889, París adquirió el monumento que se ha convertido en símbolo de la ciudad, la torre construida por Gustave Eiffel que 
servía como puerta de entrada a la feria, aparte de la remodelación de toda la zona del Campo de Marte, la construcción del Trocadero y la estación de Orsay, completadas en otras ediciones de la Exposición que tuvieron lugar en la capital francesa. Otras ediciones mundialmente famosas se dieron en Viena, en 1873 - con la participación de 35 países y 7.255.000 visitantes-, Bruselas en $1897-27$ países y 7.800.000 visitantes - y Saint-Louis en $1904-60$ países y 19.694 .855 visitantes ${ }^{1}$ - , todas ellas con repercusiones urbanísticas, en mayor o menor grado, en las ciudades anfitrionas.

Los eventos deportivos que surgieron posteriormente, la versión moderna de los Juegos Olímpicos y la Copa Mundial de Fútbol, se ubican en un periodo histórico marcado por un creciente internacionalismo político — entendido como la cooperación entre naciones - expreso en la proliferación de exposiciones, reuniones, conferencias, convenciones internacionales, que han establecido estándares y acuerdos en diversos sectores de la vida social. De acuerdo con Short (2004), el resurgimiento de los Juegos Olímpicos forma parte de las tendencias de más amplio alcance de este internacionalismo. Iniciados por el barón Pierre de Coubertin, de origen francés, los Juegos modernos se inauguraron como un proyecto político que, a través de la proyección del deporte nacional, tenía como objetivo la promoción de la paz entre las naciones. Sin embargo, inicialmente se trataba de un proyecto restrictivo y altamente elitista, ya que los atletas y delegaciones tenían que hacerse responsables de sus propios gastos, lo que únicamente hacía viable la participación de los países más ricos de Europa y de los Estados Unidos de América.

Coubertin tenía como objetivo revivir los ideales de los Juegos Olímpicos de la antigua Grecia, tal como expresaba en su filosofía acerca del nuevo Movimiento Olímpico que había creado:

Olympism is a philosophy of life, exalting and combining in a balanced whole the quality of body, will and mind. Blending sport with culture and education, Olympism seeks to create a way of life based on the joy found in effort, the educational value of good example and respect for universal fundamental ethical principles. (Rustin, 2009:3).

El aura "sagrada" de estos valores y principios sigue vigente hasta hoy; puede encontrarse especialmente en los espectáculos promovidos para la procesión de

1. Datos del Bureau International des Exposicions, responsable, desde 1928, de la regulación y fiscalización de las Exposiciones Universales. http://www.bie-paris.org 
la antorcha olímpica por distintos países, así como para las ceremonias de apertura y clausura, en las que participan miles de personas y poseen una audiencia televisiva millonaria. Sin embargo, un recorrido por algunos acontecimientos de la historia de las Olimpíadas nos enseña claramente que este evento representa mucho más que simples competiciones deportivas entre naciones.

Rubio (2005) analiza la relación entre la realización de las Olimpíadas Modernas de Verano con las transformaciones políticas y sociales del mundo, proponiendo la clasificación de sus ediciones en cuatro fases distintas: la fase de establecimiento (1896 a 1912), la fase de afirmación (1920 a 1936), la fase de conflicto (1948 a 1984) y la fase de profesionalismo (1988 hasta los días actuales). De acuerdo con la autora, las diferentes fases ilustran el paulatino crecimiento en la importancia de los Juegos en el panorama político y económico mundial, lo que se refleja en el crecimiento del calibre de la infraestructura necesaria para su realización y en su consecuente proceso de profesionalización.

El camino recorrido entre los primeros Juegos de 1896, que tuvieron lugar en Atenas, y su versión más reciente, constituye también la transición de una filosofía basada en la confraternización entre países y culturas hacia la ampliación de las condiciones de competitividad en el mercado global como objetivo a perseguir por las ciudades sede de Olimpíadas. El descubrimiento del potencial lucrativo del evento tiene como marco los espectaculares Juegos de Los Ángeles de 1932, y los de Berlín de 1936, considerados en la época un completo éxito en términos de organización y público. Los Juegos alemanes costaron unos 30 millones de dólares estadounidenses de la época al gobierno del III Reich, destinados a la construcción de estadios, piscinas y otras instalaciones deportivas. En cambio, está verificada una recaudación inédita de 3 millones de dólares, lo que junto con la experiencia de Los Ángeles cuatro años antes concretaba la idea de que las Olimpíadas podrían transformarse en un evento rentable. A partir de 1960, cuando Roma inaugura la transmisión televisiva en directo de las Olimpíadas, la venta del evento se vio impulsada en dimensiones nunca antes contempladas y, año tras año, las cifras fueron considerablemente ampliadas. Los Juegos de Tokio de 1964 fueron concebidos con la idea de representar su capacidad de superación frente a la II Guerra Mundial y su inclusión entre las principales potencias mundiales. Las inversiones de 1,8 billones de dólares en infraestructuras superaron todas las ediciones anteriores, con la creación de una importante red de servicios que quedó como legado para la ciudad. Fue en 
Los Ángeles 1984 cuando por primera vez se formó un Comité para la captación de recursos junto con la iniciativa privada, con vistas a evitar deudas, tal como se había registrado en Montreal ocho años antes. Con la inauguración de este nuevo modelo, aparte de la reducción de gastos públicos — que se limitaron a cerca del $10 \%$ del gasto total-, se produjo un beneficio de alrededor de 250 millones de dólares. Siguiendo este modelo (estadounidense) de gestión, los Juegos de Atlanta (1996) optaron por la privatización de casi la totalidad de las inversiones, mientras que en Pekín 2008 se dio una proporción contraria entre gastos públicos y privados.

Lo que aquí resulta importante señalar es la coincidencia del comienzo de la actual fase de los Juegos, la de la profesionalización, con el periodo histórico correspondiente a la globalización de la economía. La intrínseca relación de los Juegos Olímpicos — como sucede con cualquier evento mundial de proporciones más pequeñas - con el panorama político y económico global no solo se reafirma, sino que asume proporciones jamás registradas en la historia. El paulatino crecimiento en las dimensiones de la espectacularidad y en el montante de recursos movido en ocasión de la realización de unas Olimpíadas ha proporcionado una completa distorsión de los principios y valores olímpicos defendidos por Pierre Coubertin a comienzos del siglo xx. De la intención de presentarse como "celebración de la paz entre naciones por medio de la competición deportiva", el evento se ha transformado en "excusa del deporte para la celebración de la competitividad entre ciudades globales".

De acuerdo con Short (2004), los Juegos modernos son eventos "glocales", constituidos como espectáculos globales, campañas nacionales y negocios municipales. Las distintas escalas de proyección atienden a los intereses específicos de cada pieza integrante de los grupos de interés, desde lo global hasta lo local.

Los ejemplos más destacados de este nuevo momento de las Olimpíadas en cuanto oportunidad de alcanzar el estatus de ciudad global, moderna y multicultural, se han registrado con Barcelona 1992 y Pekín 2008, ejemplos exitosos de uso de los Juegos como impulsadores no solo de transformación urbana y creación de una nueva imagen para la ciudad en el contexto mundial, sino también en cuanto instrumento de afirmación de poder del país en el panorama político y económico (más específicamente en el caso de Pekín.) 
Los Juegos Olímpicos han pasado a representar una oportunidad única de realizar operaciones urbanas jamás posibles en un periodo tan corto de tiempo y en condiciones tan favorables en términos políticos y sociales. Además de la atracción de inversiones a gran escala que posibilitan estas grandes obras urbanas, entre las "condiciones favorables" está la movilización del optimismo ciudadano y del orgullo patriótico, alimentados por la alegría en tener su ciudad como anfitriona del evento deportivo más importantes del mundo.

El modelo profesional de gestión y preparación de la ciudad olímpica obedece a la misma lógica y produce los mismos resultados de la ciudad global: la intensa participación del capital privado internacional - lo que genera conflictos entre las demandas internas y externas que derivan, invariablemente, en el establecimiento de prioridades para las últimos en detrimento de las primeras-; el agravamiento de la polarización social y segregación urbana; la proliferación de instalaciones y equipamientos orientados hacia el uso de un público elitizado y temporal; o el uso del marketing como herramienta de reconstrucción de la imagen de la ciudad y establecimiento del "consenso social", por citar apenas algunos. Esta es, seguramente, una observación obvia, ya que es previsible que las dinámicas presentes en el estado actual del sistema capitalista se reflejen en esta condición de producción casi instantánea de espacios urbanos. Sin embargo, la relación existente entre el factor tiempo y la aplicación de una cantidad probablemente sin precedentes de recursos en obras de infraestructura urbana, se erige como un nuevo elemento determinante de una forma actualizada de estado de excepción, como veremos más adelante.

En un país como Brasil, en pleno proceso de emergencia económica, pero todavía con altos niveles de desigualdad social y de distribución de renta, la realización del Mundial de Fútbol de 2014 y de los Juegos Olímpicos de 2016 constituyen una gran oportunidad de cambio social. En la práctica, así como lo fueron los Juegos Panamericanos de 2007, no solo el Mundial de 2014, sino los Juegos Mundiales Militares de 2011, el Rock in Río del mismo año, la Cumbre de la Tierra Río+20 en 2012 y la Copa de las Confederaciones de Fútbol en 2013, representan importantes etapas de la preparación de la ciudad de Río de Janeiro para el objetivo mayor, que son las Olimpíadas de 2016.

Una agenda tan llena de compromisos internacionales, prácticamente anuales, forma parte de un plan de proyección masiva de la ciudad carioca y del país, lo que determina una rigidez todavía más grande en el cumplimiento de plazos. 
Es casi como si la existencia de tantos eventos funcionase como una garantía de que la ciudad estará lista para los Juegos Olímpicos, y sin los cuales la viabilidad de cada proyecto no fuera posible. Precisamente por esto, por la obligación de atender bien a todos los eventos, bajo la amenaza de la pérdida de credibilidad frente a todos los organismos internacionales involucrados, la instauración de la ciudad de la excepción se hace necesaria, urgente. Y también el apoyo de la ciudadanía, ya que en este momento de emergencia todos debemos permanecer unidos, "to celebrate nature, harmony, energy, to celebrate joy, to celebrate peace, diversity, culture, friendship, youth, hospitality, determination and excellence. To celebrate the olympic spirit" (Texto contenido en uno de los videos divulgados en la ocasión de la candidatura de la ciudad ${ }^{2}$.)

Bonito de verse, hasta diría emocionante, es otro video institucional ${ }^{3}$, también divulgado en la misma ocasión, en el cual la población común carioca - el trabajador que limpia la calle, la estudiante, una madre con su hijo, los pescadores, el vendedor callejero, los deportistas, etc. - cantan juntos la famosa canción "Cidade maravilhosa" de la ciudad, su gente, sus barrios tradicionales. Tras la emoción causada por la belleza de la pieza publicitaria, algunos se quedan extasiados y orgullosos, mientras que otros, un poco más desconfiados, pueden preguntarse: ¿serán los personajes retratados en la publicidad, de hecho, los verdaderos beneficiados con el desarrollo que los Juegos Olímpicos prometen?

\section{Patrones de la ciudad olímpica: Informe del COHRE $^{5}$}

De acuerdo con las investigaciones realizadas por la ONG suiza COHRE - Center On Housing Rights and Evictions (Centro del Derecho a la Vivienda

2. Textos contenidos en uno de los videos divulgados con ocasión de la candidatura de la ciudad. Disponible en http://www.youtube.com/watch?v=SUiSjSqhpFA\&feature=player_embedded

3. Disponible en http://www.youtube.com/watch?v=Z00jjc-WtZI

4. Canción carnavalesca compuesta por André Filho en 1935, que se hizo muy popular, convirtiéndose en un himno de exaltación de la ciudad.

5. "Fair play for housing rights". Suiza: COHRE, 2007. Disponible en www.cohre.org/mega-events.

6. Organización no gubernamental suiza, con sedes secundarias en América Latina, África y Asia, cuya misión es garantizar el pleno disfrute del derecho humano a una vivienda adecuada para todos, en todas partes. Posee publicaciones en las áreas de los desalojos forzosos, la seguridad de la tenencia, el acceso a la tierra, el 
y contra los Desalojos) - , la preparación de los JJOO acarrea, muy a menudo, que un considerable número de personas pierdan sus casas, que se deba lidiar con un aumento de la pobreza, la vulnerabilidad social y la precariedad. El problema no es nuevo; más bien sus orígenes remontan a las Olimpíadas de 1936 en Berlín, cuando el gobierno nazi, buscando la presentación de una buena imagen ante la comunidad internacional, promovió una campaña de remoción de todos los sin techo, de viviendas inadecuadas y de otras formas de pobreza en la ciudad. Desde entonces, y en crecientes dimensiones, los Juegos Olímpicos vienen significando, para las minorías desfavorecidas, un sinónimo de incremento de la pobreza, violencia, represión y pérdida de sus casas. Como resultado de ello, la contradicción existente entre los objetivos de paz, desarrollo y diálogo internacional que forman parte del discurso del Movimiento Olímpico, y la realidad truculenta vivida por algunos segmentos de la sociedad, corrompe los posibles resultados positivos de los impactos sociales generados por los Juegos en sus ciudades sede.

El estudio de las prácticas y políticas adoptadas en la preparación de los Juegos de Seúl (1988), Barcelona (1992), Atlanta (1996), Sydney (2000), Atenas (2004) y Pekín (2008) ha identificado una serie de características afines, algunas de las cuales constituyen buenos ejemplos, en mayor o menor grado, de violaciones de derechos humanos. Destacamos a continuación algunos rasgos comunes a las ciudades anfitrionas:

\section{a) Legado de mejoras infraestructurales}

El gran potencial de atracción de inversiones ofrece a las ciudades grandes oportunidades de proveer significativos legados en términos de mejoras en su infraestructura. El patrón de inversiones identificado en este campo se centra en el sistema de transporte público, el sistema viario, la construcción de aeropuertos, la remodelación de antiguas áreas industriales en proceso de degradación, las medidas de protección y mejoras ambientales y el tratamiento de espacios públicos. También se ha identificado que algunas ciudades aprovechan la ocasión para espolear, además del sector deportivo, el sector cultural, a través de la construcción de nuevos centros de cultura y arte.

agua y el saneamiento, mujeres y derecho a la vivienda, los litigios y la defensa legal de restitución y el impacto de los mega eventos en el derecho a la vivienda. Más informaciones en: http://www.cohre.org/ 
Barcelona, por ejemplo — conocida como la experiencia olímpica de mayores éxitos urbanísticos—, construyó la ampliación de su aeropuerto y un anillo viario, reformó una antigua zona industrial, abrió la ciudad hacia el mar —a través de la reestructuración del puerto-y promovió aperturas y mejoras en diversas plazas públicas. En el caso de Barcelona se dice que en los ocho años de preparación para las Olimpíadas se construyeron 50 años de infraestructura urbana.

\section{b) Efecto catalizador de desarrollo urbano}

Con los recursos financieros captados durante el periodo de la preparación de los JJOO el desarrollo que es posible alcanzar en un corto periodo de tiempo supera, en mucho, la capacidad que una ciudad puede abarcar con sus presupuestos ordinarios. Así, los Juegos también desempeñan un importante papel en la propuesta de nuevos proyectos o en la aceleración, intensificación o expansión de proyectos existentes de urbanización, elitización y planes de remodelación. Algunas veces también pueden actuar en el sentido del replanteamiento de prioridades y legitimación de planes paralizados anteriormente por motivo de conflictos con la sociedad.

En estos contextos, los límites entre el desarrollo urbano y los eventos de corto plazo se mezclan con la elaboración de una agenda compartida de prioridades en la revitalización urbana. Sin embargo, el incremento sin precedentes en la construcción civil, así como la proyección internacional que frecuentemente gana la ciudad en los años que preceden los Juegos, ejercen una presión que no siempre se resuelve en una conciliación adecuada de intereses.

En este sentido, muchas ciudades optan por revitalizar sus zonas centrales, o más bien por ubicar la mayor parte de sus instalaciones en zonas descuidadas, de bajo valor inmobiliario, que justifiquen la revitalización. En estos casos, son frecuentes los desalojos forzosos y remociones de comunidades de baja renta, cuyo poder político normalmente no es suficiente para hacer frente a la causa del embelesamiento de la ciudad.

Los estudios también señalan que en los procesos de remodelación impulsados por los megaeventos el enfoque no es interno; mejor dicho, no va dirigido necesariamente a revitalizar áreas relegadas, degradadas, pobres de la ciudad - lo que no se configura como un impedimento a que sí se haga-. Los facto- 
res externos, como el imperativo de hacer la ciudad más atractiva a la inversión del capital extranjero y al turismo, son más importantes a la hora de definir las prioridades.

Pero mucho más allá de la infraestructura necesaria para la realización de unos Juegos, las transformaciones urbanas propiciadas confieren a las Olimpíadas el estatus de herramienta para el desarrollo a gran escala. El serio problema que esto engendra es el recurrente riesgo del redireccionamiento de las políticas públicas en atención a las prioridades determinadas por el comité de organización, los cuales tienen pleno poder de decidir los rumbos de la ciudad olímpica.

\section{c) Colaboración entre grupos de intereses}

La realización de las Olimpíadas demanda un alto (y muchas veces inédito) nivel de cooperación entre las autoridades estatal y local, y/o entidades privadas, lo que debe resultar, necesariamente, en la "fabricación" de un consenso. La planificación colectiva de las acciones en el ámbito de la ciudad olímpica demanda, por lo menos en apariencia, una convergencia de intereses, a veces imposible.

Por otro lado, la cooperación del Estado con el capital privado, las famosas asociaciones público-privado, se hace de extrema necesidad una vez que las operaciones urbanísticas tienen costes insostenibles para las arcas públicas.

\section{d) Efectos nocivos en grupos ya marginados y vulnerables}

Otra característica común a las ciudades sede de las Olimpíadas es la concentración de los beneficios proporcionados por las inversiones en manos de las clases ya privilegiadas. La modernización y el embellecimiento necesarios al contexto olímpico traen consigo el agravamiento de la situación de aquellos que ya viven en las condiciones más desfavorecidas, que son los principales afectados en todo el proceso. No solo en los casos de expropiaciones, desalojos forzosos y desahucios con vistas a la construcción de instalaciones o infraestructura necesaria para la realización de los Juegos, sino también porque la valorización del suelo urbano promueve la expulsión de la población habitante de áreas cuyos costes se elevan exageradamente. Si por un lado ganan los propietarios, por otro pierden los inquilinos, obligados a marcharse hacia zonas más lejanas del centro urbano. 
Las frecuentes violaciones de los derechos humanos incluyen operaciones de "limpieza social", que eliminan los sin techo del paisaje urbano antes y durante los Juegos, acompañado de su criminalización. También se repite la intensificación de las acciones policiales en comunidades de baja renta, con restricciones de libertades civiles.

En todos los casos estudiados se encontraron ejemplos de acciones de violencia directa e indirecta hacia las poblaciones en situación de vulnerabilidad social.

\section{e) Mentalidad de un "estado de excepción"}

Bajo la excusa de la realización de las Olimpíadas, que se contempla como una oportunidad única que debe ser aprovechada de la manera más rentable posible, se admite la adopción de prácticas y políticas que difícilmente serian aceptadas en otras condiciones. Por parte de la sociedad, los niveles de tolerancia se tornan bastante más flexibles, mientras que por parte de los organizadores, la circunstancia de excepcionalidad les permite llevar a cabo decisiones polémicas, restricciones de derechos, uso de violencia y otras medidas extremas que se consideran "necesarias" para la empresa olímpica. En este sentido, también las legislaciones suelen ser fruto de adaptaciones con vistas a viabilizar proyectos y operaciones millonarias llevadas a cabo en tan corto plazo, como legitimar medidas excepcionales con relación a la reducción de derechos de inquilinos o la criminalización de los sin techo, entre otras socialmente inaceptables.

De acuerdo con Carlos Vainer (Vainer, 2011), este hecho se configura como una actitud propia del pensamiento fragmentado posmoderno y debe ser comprendido como una estrategia de poder, basada en la idea de flexibilización y en la sustitución de la noción de interés común por la legitimación de la multiplicidad de intereses. En un análisis de los clásicos marxistas, de Marx a Poulantzas, el autor señala que todas las formas totalitarias de poder por ellos estudiadas conformarían un "estado de excepción", que se configura como un tipo de régimen en el cual las clases dominantes no logran asumir directamente las riendas del poder y, en cierta manera, delegan la dirección del Estado a un "bonaparte" o a un grupo particular (militares) (Vainer, 2011: 9). En tiempos 
de globaritarismo, o quizás sea más adecuado decir "en tiempo de globalismo"7, en lugar de militares, una parte importante de las funciones del Estado es regulada y ejercida por el mercado, por el capital privado.

A cidade da exceção se afirma, pois, como uma nova forma de regime urbano. Não obstante o funcionamento (formal) dos mecanismos e instituições típicas da república democrática representativa, os aparatos institucionais formais progressivamente abdicam de parcela de suas atribuições e poderes. A lei torna-se passível de desrespeito legal e parcelas crescentes de funções públicas do estado são transferidas a agências "livres de burocracia e controle político" (Vainer, 2011: 10).

Para Vainer, los megaeventos hacen de la "ciudad de la excepción" una realidad plena e intensa. La emergencia del cumplimiento de un calendario limitado e inalterable es la razón que justifica la infracción de la legislación urbanística, la supresión de la participación popular, la opresión de los movimientos sociales. Adicionalmente, la diseminación de las ideas de embellecimiento y revitalización necesarios al hospedaje de los JJOO sirven de base para la conquista de la aprobación y el consenso de la sociedad.

\section{f) Constante búsqueda del apoyo de la comunidad}

Este punto es, precisamente, lo que hace posible la instauración del estado de excepción tratado anteriormente. La aprobación de la población, conseguida a través de la movilización del orgullo patriótico, se configura como una condición necesaria para el éxito de los Juegos. Así, con vistas a minimizar los impactos negativos de las Olimpíadas, se suele juzgar la actitud de los movimientos de oposición como un comportamiento pesimista y contrario al desarrollo de la ciudad.

En este constante proceso de seducción de la población —uno de los requisitos exigidos por el Movimiento Olímpico durante los ocho años de preparación de los Juegos - son frecuentes las campañas publicitarias masivas, cuya apelación emocional se centra, con frecuencia, en la esperanza de cambio y en el orgullo de una identidad construida artificialmente, y la movilización de can-

7. Santos (2000) se refiere a globaritarismo como una versión actualizada de totalitarismo en tiempos de globalización. Ya el concepto de globalismo, de la manera como lo propone Beck (2000), engloba la idea de la adopción del mercado mundial como sustituto del poder del Estado. 
didatos al voluntariado, importante termómetro de la eficacia de las maniobras de marketing.

\section{g) Limitaciones en la participación social, consulta comunitaria y transparencia}

La ausencia o escasez de estos elementos en la mayor parte de las experiencias estudiadas es una constante que conecta directamente con el carácter en general despolitizador de los megaeventos. La reducción de la participación social a lo mínimo posible funciona no solo como estrategia de defensa de intereses ajenos a la población, sino también como simplificación del proceso decisorio con vistas a atender al calendario de los Juegos. La magnitud de las decisiones y cifras movidas, al parecer, suele ser incompatible con el modelo de participación hasta hoy experimentado en las ciudades sede de Olimpíadas. El largo tiempo que necesitan los procesos de consulta y decisión compartida, añadido a la incapacidad de alcanzar un consenso real, hacen de ese tema un punto de conflicto permanente en el contexto olímpico.

\section{Un futuro muy cercano: Londres 2012}

En julio de 2005, en una sesión celebrada en Singapur, la ciudad de Londres fue elegida como sede de los XXX Juegos Olímpicos, derrotando a las contrincantes París, Madrid, Moscú y Nueva York, y convirtiéndose así en la primera ciudad del mundo en albergar las Olimpíadas por tercera vez (1908, 1948 y 2012). Desde entonces se ha puesto en marcha el proceso de preparación de la sede olímpica, cuya inauguración se dará con la apertura de los Juegos, que se disputarán del 27 de julio al 12 de agosto de 2012.

Teniendo como uno de sus principios fundamentales la restricción de las construcciones al mínimo imprescindible, el proyecto de Londres 2012 fue estructurado para evitar los "elefantes blancos" - a ejemplo de su Millenium Dome, construido para una exposición en 2000 y desde entonces infrautilizado- y centrar el legado olímpico en la forma de remodelación e inserción urbana de una antigua zona industrial del este de Londres. Más que una celebración deportiva, los Juegos serán, aquí, sinónimo de regeneración.

Si lo comparamos con el proyecto de Pekín 2008, el Master Plan del Parque Olímpico de Londres se muestra más compacto y de dimensiones más modes- 
tas: en total, ocupa una superficie de 240 hectáreas en el Lea River Valley (Valle del río Lea), mientras que en los Juegos de 2008 se ocupó un total de 1.215 hectáreas, de las cuales 760 fueron destinadas a la implementación de zonas verdes y boscosas.

Una de las principales características de la implantación de las instalaciones olímpicas es la opción de concentrarlas alrededor de una misma zona, en el distrito de East London, en la región conocida como Lea Valley, dentro de los límites del Gran Londres. El Parque Olímpico se localiza en un área ocupada por cinco municipios (Hackney, Newham, Tower Hamlets, Waltham Forest y Greenwich), cuya porción más afectada se ubica en el distrito de Stratford, municipio de Newham. Se extiende por las márgenes del río Lea, en puridad un brazo del río Támesis, y ocupa un área definida por la separación entre el tejido urbanizado más cercano a Londres y su zona periférica al este, una antigua zona industrial.

Aunque los Juegos se extiendan por otras zonas de la ciudad y por los alrededores del Gran Londres, será en el Parque Olímpico donde se centrarán los eventos más importantes y donde se reúnen las principales instalaciones y legados posolímpicos, tales como el Estadio Olímpico, el Centro Acuático de Londres, el Velopark, el Centro Olímpico de Hockey, la Villa Olímpica, el Centro Principal de Prensa (MPC) y el Centro Internacional de Radio y Televisión (IBC), entre otras instalaciones de carácter temporal.

La preocupación por el tema de la participación social y la transparencia se refleja en el establecimiento de una serie de compromisos y declaraciones públicas, constituidos a través de consultas a la sociedad. A partir de estos instrumentos, la comisión organizadora prevé el uso del control social como herramienta indicativa del éxito de los Juegos. El objetivo es paliar algunos de los principales problemas identificados en ediciones anteriores y convertirse así en una referencia para la realización de unas olimpíadas, tal como afirma el alcalde de Londres, Boris Johnson, en un evento realizado recientemente en la Plaza Trafalgar: "Serán los mejores Juegos Olímpicos de la historia del deporte".

8. Disponible en la página web: http://esportes.terra.com.br/rumo-a-2012/noticias/0,,OI5264450EI17396,00-Londres+faz+festa+e+promete+melhor+Olimpiada+da+historia.html 


\subsection{Regeneración Urbana}

Whereas the Beijing Games celebrated a dynamic economy and a society announcing its entry onto the world stage, the London Games reflects the aspirations of a less dynamic economy and society for whom 'regeneration' consists of addressing a lengthy period of under-investment in the infrastructure and social development of its capital city, particularly in a part of the city that has long been neglected. (Poynter, 2009: 197).

Este fragmento del texto de Poynter señala una diferencia básica entre el enfoque dado a los Juegos de 2008 en Pekín y el proyecto de Londres 2012: mientras el primero hizo uso del evento como una excusa para su proyección mundial en cuanto potencia económica emergente - reflejada en gastos exorbitantes y estructuras megalómanas-, Londres ha optado por centrar los beneficios ofrecidos por los Juegos en la regeneración y recuperación de una región degradada.

La elección de una antigua zona industrial y de servicios, ahora absorbida por el tejido urbano, refleja el desplazamiento del interés del poder público, desde hace ya más de treinta años, hacia la zona este de la ciudad. Desde 1981, East London viene siendo objeto de algunos proyectos emblemáticos de regeneración urbana, tales como los realizados en los Docklands y el Thames Gateway.

El primero de éstos fue llevado a cabo por la London Docklands Development Corporation (LDDC), que, entre 1981 y 1998, promovió la urbanización de áreas degradadas en los Docklands, abarcando un área de $22 \mathrm{~km}^{2}$ en los municipios de Newham, Southwark y Tower Hamlets con la construcción de 24.402 viviendas (Bernstock, 2009).

El proyecto del Thames Gateway ("entrada del Támesis"), llevado a cabo en una extensión de $60 \mathrm{~km}$ en ambas márgenes del río Támesis, y que engloba 16 distritos industriales entre las regiones de London, East y South East -incluyendo el área del actual Parque Olímpico-, se configura como la implementación de 106 acuerdos relacionados con el Plan de desarrollo municipal, con vistas a conciliar el provecho del sector privado con beneficios para las comunidades locales. El área posee una población de alrededor de 1,6 millones de personas y contiene algunos de los distritos más pobres del país, que se caracterizan por la falta de acceso al transporte público, de servicios, de empleo y por la vivienda de baja calidad. En marcha desde 1981, los acuerdos buscan, 
al mismo tiempo, incentivar inversiones en el desarrollo urbano - a través de concesiones - y reducir los impactos sociales de iniciativas privadas - a través de la garantía de contribución en infraestructura-, construyendo nuevas vías y escuelas y, en algunos casos, creando nuevos espacios públicos, contribuyendo a la creación de centros de juventud y ofreciendo garantías de acceso a oportunidades de trabajo generadas por la construcción. Los acuerdos también incluyen la especificación de una proporción de casas asequibles para los sectores de población más pobres, si bien su cumplimiento es objeto de críticas por parte de algunos especialistas.

\subsection{Legado postolímpico}

El proyecto olímpico de Londres 2012 fue pensado de manera que supusiera la continuación de las iniciativas de regeneración en marcha en East London, sobre todo en Lea Valley. El legado en términos de infraestructura urbana incluye el soterramiento de cables de electricidad, una compleja red de conexiones viarias y la urbanización de las márgenes del río Lea, conectando la superficie ya urbanizada del río Támesis creando un espacio público lineal y continuo, del centro de la ciudad a la zona periférica.

El Parque Olímpico será construido en dos niveles, el del río y otro superior, una suerte de plataforma donde se ubicarán las instalaciones. Después de los Juegos, algunas de las áreas pavimentadas serán revestidas con césped y árboles; así, las áreas pensadas para el uso simultáneo de millares de personas durante el evento se adecuarán al uso de la población local a través de su transformación en un gran parque. La preocupación con respecto a la infrautilización de las instalaciones se refleja en el gran número de estructuras efímeras que se han proyectado. Entre todas las instalaciones deportivas construidas solamente cuatro serán permanentes - el estadio principal, el centro acuático, el pabellón de balonmano y el velódromo - y se sumarán al parque integrándose al distrito que las rodea para que sirvan como equipamiento comunitario.

El Estadio Olímpico de Londres, principal estadio de los Juegos, tiene una capacidad inicial para 80.000 espectadores gracias a la construcción de un anillo temporal, cuya retirada después de los Juegos le reducirá la capacidad hasta un máximo de 25.000 personas. La idea es que dos tercios de sus estructuras puedan ser reutilizadas después del desmontaje. También los asientos se reuti- 
lizarán en diferentes instalaciones británicas, y la carpa blanca de PVC viajará a los Juegos Olímpicos de Río de Janeiro de 2016.

El desmontaje de las estructuras temporales y posterior preparación del legado permanente ocupará un tiempo prolongado: se prevé su conclusión solo en el verano de 2013. Después de los Juegos, las instalaciones del Centro de Prensa IBC/MPC darán lugar a la creación de $80.000 \mathrm{~m}^{2}$ de espacios comerciales, con capacidad para generar miles de puestos de trabajo.

En cuanto a los servicios de transporte, la zona - ya conectada anteriormente por tres líneas de metro, dos líneas de tren, una extensión del tren ligero Docklands Light Railways y una estación del Eurostar - tendrá su red ampliada y contará con la implantación de un nuevo servicio de tren de alta velocidad, llamado Javelin. Aparte, se instalará un sistema de transporte por teleféricos sobre el río Támesis, entre la península de Greenwich y los Royal Docks, con capacidad para transportar 2.500 personas por hora en cada dirección, lo que corresponde a la capacidad total de 50 autobuses.

Más allá de la regeneración urbana, uno de los retos que afronta la comisión de Londres 2012 es la capacidad de catalizar la regeneración social del East London. El proyecto olímpico asume compromisos con la sostenibilidad a través de la creación de puestos de trabajo, programas orientados al entrenamiento deportivo y la capacitación profesional y ocupándose de asuntos como la vivienda y el medio ambiente. Sin embargo, todavía es demasiado temprano para evaluar la eficacia y los impactos más duraderos de estos programas.

\subsection{Anticipación y ahorro}

Hasta el cierre de este texto, la experiencia de Londres apuntaba hacia la elevación de algunos patrones en la preparación de una ciudad anfitriona, sobre todo en términos de cumplimiento del calendario y reducción de gastos.

Declaraciones oficiales realizadas el 19 de julio de $2011^{9}$ confirmaban la conclusión del 88\% de las obras previstas para los Juegos Olímpicos de 2012. De acuerdo con el profesor de marketing deportivo Alan Seymour ${ }^{10}$, de la Uni-

9. Disponible en: http://www.estadao.com.br/noticias/geral,obras-de-olimpiadas-de-2012-em-londresestao-88-prontas-diz-comite,746929,0.htm

10. En entrevista publicada en http://www.valoronline.com.br/impresso/especial/101/463229/sem-atrasos-londres-tera-a-sua-olimpiada-dentro-do-orcamento. 
versidad de Northampton (Reino Unido), resulta inédito tener una sede olímpica casi lista con doce meses de antelación.

Aparte de la ya conocida puntualidad británica, la agilidad en el proceso constructivo se consiguió al optar por el uso de estructuras temporales y por aprovechar infraestructura ya existente, como por ejemplo el uso del área del Horse Guard's Parade (el desfile de caballos de la Guardia Real) para el voleibol playa, el Greenwich Park para la hípica y el estadio de Wembley para el fútbol. Estas elecciones han aportado importantes ahorros. Aunque el presupuesto final haya sufrido un polémico incremento correspondiente a cuatro veces el valor inicialmente previsto - pasando de 2.400 millones de libras esterlinas a 9.300 millones-, los gastos serán notoriamente más bajos que los emprendidos en las Olimpíadas de Pekín en 2008 (más de 40.000 millones de dólares USA) y muy próximos al presupuesto final de Atenas 2004 (alrededor de 14.000 millones de dólares USA).

En términos de financiación, a diferencia de Atenas y Atlanta el poder público ha sido el responsable mayoritario en la financiación del evento, cuyas inversiones se suman a los recursos provenientes del Comité Olímpico Internacional (COI) y de los patrocinadores, e incluyen fondos procedentes de la lotería estatal. De esta manera, se ha asegurado un mayor compromiso con la responsabilidad social del legado de los Juegos.

Los mayores desafíos de la comisión organizadora se relacionan con el tema de los transportes - el talón de Aquiles de la ciudad, sobre todo por la limitación de la anticuada red de metro- - y con la seguridad - ya que el país mantiene un nivel "severo" de alerta hacia la amenaza terrorista.

\section{Consideraciones finales}

Naturalmente, no todas las enseñanzas útiles que se pueden extraer de ciudades olímpicas previas a los Juegos de 2016 son aplicables a la realidad de una ciudad brasileña. Por ejemplo, el ahorro en estructuras verificado en Londres deriva, en parte, del hecho de que sea ya la tercera edición de las Olimpíadas que va a realizarse en la ciudad, lo que les confiere la ventaja de poseer ya algunas importantes instalaciones, aunque sean necesarias algunas reformas. Sin embargo, la preocupación londinense de no dejar "elefantes blancos" - fruto de su experiencia propia y de la observación de otras ediciones de los Juegos- es 
un importante aspecto que puede y debe ser observado, no solo por la ciudad carioca, sino también por las ciudades brasileñas que serán sede de los juegos del Mundial de Fútbol 2014, cuyos estadios ya se encuentran en proceso de reforma/reconstrucción de acuerdo con los patrones de la Federación Internacional de Fútbol Asociación (FIFA). En Pekín, por ejemplo, el estadio olímpico, conocido como "nido de pájaros", sigue hasta hoy en situación de infrautilización, lo que lo configura como un "juguete" de casi 350 millones de euros. En Sudáfrica, el estadio conocido como Soccer City, en Johannesburgo, aunque presente características más modestas que el estadio chino, es criticado por su inadecuación a su contexto socioeconómico. El exceso de aparatos dedicados al control y seguridad - equipamientos modernos de lectura electrónica, detectores de metal, cámaras de seguridad - hace necesarios altos costes de mantenimiento, que se reflejan en los precios de las entradas, incompatibles con la realidad local.

Tanto en las experiencias del Parque Olímpico de Londres como en la Villa Olímpica y zona portuaria de Barcelona se observa la prioridad de inversión en la renovación y reinserción de antiguas áreas degradadas, devueltas a la ciudad. Aunque en el ejemplo barcelonés el uso posterior de las unidades residenciales de la Villa haya sido revertido para el mercado inmobiliario, son innegables los beneficios urbanísticos que esa nueva zona ha aportado para los ciudadanos; lo mismo para el área del Port Vell, que, al mismo tiempo que concentra restaurantes, bares y equipamientos de ocio de uso privado, ofrecen un espacio público de calidad para el uso colectivo e irrestricto. Así, algunas experiencias enseñan que las inversiones propiciadas por los Juegos, utilizadas la renovación urbana y la producción de espacio público, pueden ofrecer buenos legados para los ciudadanos en general.

Sin embargo, la elitización provocada por las inversiones olímpicas es un asunto todavía sin solución en la preparación de las ciudades anfitrionas. En todos los ejemplos ilustrados se ha podido observar la expulsión de la población residente en las zonas afectadas por obras relacionadas con los Juegos, sea por desahucios forzosos, por realojamientos o por encarecimiento en el coste de vida. La observación de estos hechos hace necesaria una atención más cuidadosa acerca de los efectos sociales de las Olimpíadas. En los estudios publicados por el COHRE se pueden encontrar números preocupantes acerca de la población desalojada, mucha de ella sin garantías de realojamiento o mejoras en las 
condiciones de vida. ¿Hasta cuándo se reproducirá este tipo de comportamiento en la organización de megaeventos? Los efectos nocivos a grupos ya marginados se configuran como una constante en los procesos de preparación de las ciudades olímpicas. Con relación a eso, ¿qué esperar de unos Juegos realizados en una ciudad donde la mayoría de la población integra esta clasificación social?

Desde la década pasada, cuando la exigencia del "legado" se instituyó en el ámbito de las Olimpíadas, lo que se observa es que en la misma proporción que esta palabra gana importancia en el contexto de la organización de los Juegos, crecen las oportunidades de captación de inversiones privadas y disminuye el compromiso social de las acciones programadas. Los elevados números de población desalojada en Seúl y en Pekín (720.000 y 1.250.000 personas, respectivamente), dos ediciones indiscutiblemente orientadas hacia la proyección de las ciudades en el escenario global, confirman esta relación inversamente proporcional entre inversión privada y compromiso con la población habitante. En el globalismo llevado a cabo por las manos de los agentes del mercado mundial, la tarea del poder público pasa a ser la de facilitador de las grandes operaciones inmobiliarias necesarias para la ampliación de las condiciones de competitividad de las ciudades globales. En el ámbito de la preparación de una ciudad para los Juegos Olímpicos, el estado de excepción instituido por el gobierno es lo que permite las sucesivas violaciones de los derechos humanos, repetidas edición tras edición; las Olimpíadas vuelven las dinámicas derivadas de la mercadotecnia urbana todavía más urgentes y justificables. Y, por supuesto, también se justifican las pequeñas alteraciones en la legislación local (a veces no tan modestas), que se convierten en una extrema necesidad para la materialización del sueño olímpico: se aprueban construcciones mucho más allá de lo permitido usualmente, poniendo el suelo urbano y los recursos públicos a servicio de los inversores privados; se permite la proliferación de "elefantes blancos" en el paisaje urbano, y se consiente la elitización de las zonas de intervención, por citar apenas algunos efectos.

Frente a las tendencias aquí observadas, según parece una cuestión clave en la práctica del actual modelo de gestión de las Olimpíadas — que aplaude la proliferación de estructuras megalómanas- es el hecho de que no solamente la viabilidad de la construcción de la ciudad olímpica, sino también la sostenibilidad de tales instalaciones en el periodo posevento, esté cada vez más vinculada 
a la comercialización de su uso. Así lo dice el pensamiento único y es lo que se viene estableciendo como norma.

Londres subvierte esta dinámica e innova cuando, aparte del ahorro en construcciones, propone el uso de estructuras efímeras que pueden ser desplazadas hacia otros eventos. En la edición de 2012 se encuentran algunos gérmenes de lo que podría entenderse como un punto de inflexión en el uso de los Juegos como instrumento de aceleración de la mercadotecnia urbana. Sin embargo, el hecho de que cada país tiene un perfil y demandas específicas se refleja en la estrategia urbana a la cual se vincula la oportunidad de albergar las Olimpíadas, de manera que no se puede esperar un desarrollo lineal en el modelo de gestión aquí identificado como hegemónico.

Finalmente, y volviendo la atención hacia los juegos brasileños, este breve panorama - aunque pueda parecer un tanto duro - no tiene la intención de constituirse como una profecía desalentadora. Más bien pretende establecerse como alerta hacia los riesgos y tendencias ya identificados en estudios anteriores, comprendiendo que la repetición de los patrones abordados solamente puede ser interrumpida a través de pequeños y sucesivos cambios de actitud frente al modelo vigente, como se ha podido ver tímidamente en el caso de Londres. Desafortunadamente, hasta que esto ocurra de hecho, no hay que dejar de cuestionarse qué costes sociales se pueden esperar de megaeventos organizados en un país de economía emergente donde, todavía en 2008, el 10\% de la población más rica detentaba el 75,4\% de todas sus riquezas. Que me perdonen los optimistas... 


\section{Referencias bibliográficas}

Allies, B. (2010) Cultivating the City: London before and after 2012. Ámsterdan: Sun.

Bеск, U. (1998) ¿Qué es la globalización? Falacias del globalismo, respuestas a la globalización. Barcelona: Paidós.

Bernstock, P. (2009) “London 2012 and the Regenerating Game”. En: Poynter, G.; Macrury, I. Olympic Cities: 2012 and the remaking of London. Londres: Ashgate. 201-218.

COHRE. Centre on Housing Rights and Evictions. (2007) Fair play for housing rights. Suíça: COHRE. Disponible en: www.cohre.org/mega-events. Acceso en 25 de julio de 2011.

DíAz, A. (2007) “En torno a la mercadotecnia urbana: reorganización y reimaginación de la ciudad”, Biblio $3 W$ (en línea), Vol. XII, n 712. Disponible en: <http://www.ub.edu/geocrit/b3w-712.htm> Acceso en 28 de agosto de 2011.

Geyer, H.S. (2002) “On urban systems evolution”. En: GEYER, H. (dir*). International Handbook of Urban Systems. Studies of Urbanization and Migration in Advanced and Developing Countries. Cheltenham, Reino Unido: Edward Elgar.

Poynter, G. (2009) "The Evolution of the Olympic and the Paralympic Games 1948-2012". En: Poynter, G; Macrury, I. Olympic Cities: 2012 and the Remaking of London. Londres: Ashgate. 23-41

—."London: Preparing for 2012". En: Poynter, G; Macrury, I. Olympic Cities: 2012 and the remaking of London. Londres: Ashgate. 183-199.

Rubio, K. (2005) "Os Jogos Olímpicos e a transformação das cidades: os custos sociais de um megaevento", Scripta Nova (en línea), Vol. IX, no 194. Disponible en: < http://www.ub.edu/geocrit/sn/sn-194-85.htm> Acceso en 30 de agosto de 2011.

Rustin, M. (2009) "Sport, Spectacle and Society: understanding the Olympics". En: Poynter, G; Macrury, I. Olympic Cities: 2012 and the Remaking of London. Londres: Ashgate. 3-21.

Santos, M. (2000) Por uma outra globalização: do pensamento único à consciência universal. Rio de Janeiro: Record. 
Sassen, S. (1999) La ciudad global: Nueva York, Londres, Tokio. Buenos Aires: Eudeba.

-. (2009) "La Ciudad Global: introducción a un concepto". En: BBVA (org.) Las múltiples caras de la globalización. Disponible en: <http://www.bbvaopenmind.com/book/las-multiples-caras-de-la-globalizacion/> Acceso en 28 de agosto de 2011.

Short, J.R. (2004) "Going for Gold: Globalizing the Olympics, Localizing the Games". En: Global Metropolitan: Globalizing Cities in a Capitalist World. London: Routledge. 86-108.

Short, J.R.; Breitbach, S.; Buckman, S.; Essex, J. (2000) “From world cities to gateway cities: Extending the boundaries of globalization theory", Cities, vol. 4, no 3:317-340.

Solomon, G. (2003) “Staged Cities: Mega-events, Slum Clearance and Global Capital". En: Yale Human Rights and Development Law Journal, Vol. 6: 161-187.

VAiner, C. (2011) Cidade da exceção: reflexóes a partir do Rio de Janeiro.. Disponible en: < http://pfdc.pgr.mpf.gov.br/atuacao-e-conteudos-de-apoio/ publicacoes/direito-a-moradia-adequada/artigos/cidade-de-excecao-carlos-vainer>

- (2000) "Os liberais também fazem planejamento urbano? En: Arantes, O.; Vainer, C.; Maricato, E. A cidade do pensamento único. Desmanchando consensos. Petrópolis: Vozes. 105-119.

Wallerstein, I. (1984) The Politics of the World Economy: The states, the movements and the civilizations. Cambridge, Reino Unido: Cambridge University Press. 